Syntactic representations in late learners of a second language: a learning trajectory

\title{
Sarah Bernolet
}

$\&$

Robert J. Hartsuiker

Corresponding author:

Sarah Bernolet

Grote Kauwenberg 18

2000 Antwerp

Belgium

sarah.bernolet@uantwerpen.be 


\begin{abstract}
Several studies have shown that syntactic structures can be primed between the different languages of a bilingual. Bilingual production models put forward by Hartsuiker, Pickering and Veltkamp (2004) and Pickering and Hartuisker (2008) therefor assume that bilinguals share syntactic structures between languages as much as possible. In this paper, we discuss a model for the development of these shared syntactic structures in late learners of a second language (Hartsuiker \& Bernolet, 2016). More specifically, we discuss evidence for three central claims of the model, namely that 1) L2 syntactic representations move from being item-specific to being more abstract, 2) L2 representations become more and more integrated with existing L1 representations, and 3) L1 influences on syntactic processing and production in the L2 occur in early and late phases of L2 syntactic development.
\end{abstract}


Acquiring the syntax of a language is a very complex and multi-faceted process: A learner needs to learn the meaning of lexical items, form syntactic categories, and distill the complete set of rules and processes that govern the structure of sentences from the input in order to learn how syntax reflects the relation between the different elements in a sentence. Children learn their native syntax (or syntaxes, if they are native bilinguals) quite rapidly, and without explicit instruction: In a year's time, their utterances evolve from twoword phrases like "Bye-bye daddy" and "Doggy sit" to complex sentences as "Can I have some milk, please, mommy?". For late learners of a second language, the acquisition of syntax is a stumbling block: It requires much effort and attention, and often some kind of explicit instruction. The learning context is in some cases very different for native syntax learning and the late acquisition of second language (L2) syntax: While native syntax is picked up implicitly from interactions with speakers in the child's direct environment, second language syntax can be formally taught when children learn a foreign language at school. Another important difference between native syntax learning and late learning of second language syntax is that in the latter case, the syntax of the first language (L1) is already fully acquired. General knowledge of syntactic concepts and systems might aid second language learning, but it could also hinder learners. The knowledge of L1 syntax might lead to 'educated guesses' for the comprehension and production of L2 syntax, which might require more conscious thought and effort than the 'trial-and-error-processing' that characterizes the acquisition of L1 syntax. Additionally, late learners might not as feel free as children do to produce ill-formed sentences in the process of learning. How do syntactic representations in the second language develop in these late learners and what is the relation between the L1 and L2 syntax? We try to answer these questions by presenting a theoretical model for the acquisition of $L 2$ syntax in late learners of a second language (Hartsuiker \& Bernolet, 2016) and discussing the different studies that led to the formulation of this model.

A model of the trajectory of late $L 2$ syntactic acquisition The production model we proposed in Hartsuiker and Bernolet (2015) is an elaboration on the bilingual production models of Hartsuiker, Pickering and Veltkamp (2004) and Pickering 
and Hartsuiker (2008). Hartsuiker et al. (2004) discovered that syntactic structures can be primed between both languages of a bilingual: Spanish-English bilinguals were more likely to describe pictures in English (L2) using passives when they had just processed a Spanish (L1) passive prime sentence (El camión es perseguido por el taxi [The truck is being chased by the taxi]), than when they had processed Spanish actives (EI taxi persigue el camión [The taxi chases the truck]) or intransitive sentences (El taxi acelera [The taxi accelerates]). Hartsuiker et al. (2004) concluded that Spanish-English bilinguals use the same syntactic rules when comprehending and producing Spanish or English passives and activate the same memory representations from both languages. Hence they proposed a lexical-syntactic model for bilingual sentence production in which syntactic information is shared between languages as much as possible. In their model, which is based on Pickering and Branigan's (1998) model of lexico-syntactic representations, information about syntactic structures is represented in combinatorial nodes, which are connected to lemma nodes in a single integrated lexicon. Thus, the lemmas of all Spanish and English transitive verbs are connected to a shared categorical node indicating that these verbs can be combined with two noun phrases in order to form a passive sentence.

In the last few years, numerous studies have shown between-language syntactic priming using a variety of language pairs, constructions, and paradigms (Bernolet, Hartsuiker \& Pickering, 2012; Cai, Pickering, Yan \& Branigan, 2011; Kantola \& Van Gompel, 2011; Salamoura \& Williams, 2006, 2007; Schoonbaert, Hartsuiker \& Pickering, 2007; Shin \& Christianson, 2009; 2012), suggesting that syntactic representations are indeed shared between languages as much as possible. Pickering and Hartsuiker (2008) made the additional assumption that, according to a lexicalist model with fully shared syntax, priming between languages should be of comparable magnitude to priming within a language, because both languages would use one and the same node. In our 2015 paper we argued that this is probably not true for all bilinguals (Hartsuiker \& Bernolet, 2015). Hartsuiker et al.'s (2004) and Pickering and Hartsuiker's (2008) models might capture important aspects of syntactic representations in native bilinguals, who simultaneously developed syntactic representations in both of their languages, and for syntactic representations in late learners of a second languages that have reached a very high level of $L 2$ proficiency. These bilinguals can be expected to show abstract structural priming of comparable magnitude within- and across-languages. For bilinguals who did not yet reach this level of $L 2$ proficiency, we expect 
between-language priming to be weaker than within-language priming, because their L2 syntactic representations are still under development. In order to be able to make predictions about the L2 syntactic output that can be generated by L2 learners and about the strength of within and between-language priming through the development of L2 syntax, we proposed a developmental model with 5 consecutive stages. In our model, learning is determined by two principles, namely representational specificity and economy: The learning system aims to capture all relevant differences between linguistic representation, but, at the same time, it tries to minimize the number of representations by sharing what can be shared. We assume that explicit memory processes play a large role in the early phases of acquisition, and that longer term learning is caused by Hebbian learning or by the implicit learning of syntactic structures (Chang, Dell, \& Bock, 2006).

The different stages of our developmental model for the representation of L2 syntax are depicted in Figure 1. The model shows the learning trajectories for the English s-genitive (s-gen: the boy's doll) and the English post-modified noun phrase (N RC: the ball that is red), structures that can both be combined with the nouns pop [doll] and bal [ball]. For simplicity's sake, the model only shows two lexical nodes and two combinatorial nodes, while leaving out the other nodes to which these nodes are connected (e.g., the conceptual nodes, the language node, the word category nodes, etc.). The representations of the English of-genitive and the English pre-modified noun phrase are also left out, though we assume that these develop together with their syntactic alternatives, the English s-genitive and post-modified noun phrase. Given that the model represents the stages of late L2 syntactic acquisition, the L1 syntax is already fully represented in the first panel of the figure, which represents the initial stage of acquisition for both structures. We assume that, in this initial stage, L2 acquisition begins with learning of lexical representations without firm connections to syntactic information. A Dutch learner of English as an L2 might have learned simple nouns like 'doll' and 'boy', but might not know which English constructions can be used to express that the doll is owned by the boy (the doll of the boy/the boy's doll). If this learner wants to express a possessive relation between both nouns, she can only rely on her knowlegde of L1 genitive structures to do the job. Transferring the L1 syntax can lead to transfer errors such as "It's the doll from the boy (Het is de pop van de jongen)" and "It's the boy his doll (Het is de jongen zijn pop)", but also to correct productions "It's the doll of the boy (Het is de pop van de jongen)". Another strategy that learners can use at this stage, 
is to imitate structures that are produced by more proficient speakers. Production is in this case rather reproductive than creative: A question like "Shall I wear the red dress or the blue one?" is likely to elicit parallel responses like "the blue one" and "the red dress", but no creative response like "the red one". We assume that these imitations are based on the retrieval of the example sentences from explicit memory. Hence, these repeated or minimally edited L2 structures only appear quickly after the learner perceived an example sentence. To summarize, in this initial stage of the acquisition of L2 syntax production is characterized by L1 transfer and primed L2 structures in immediate conditions when there is a high lexical overlap between consecutive sentences. Comprehension of L2 syntax is also guided by L1 syntactic preferences at this stage, as no L2 syntactic representations are available yet.

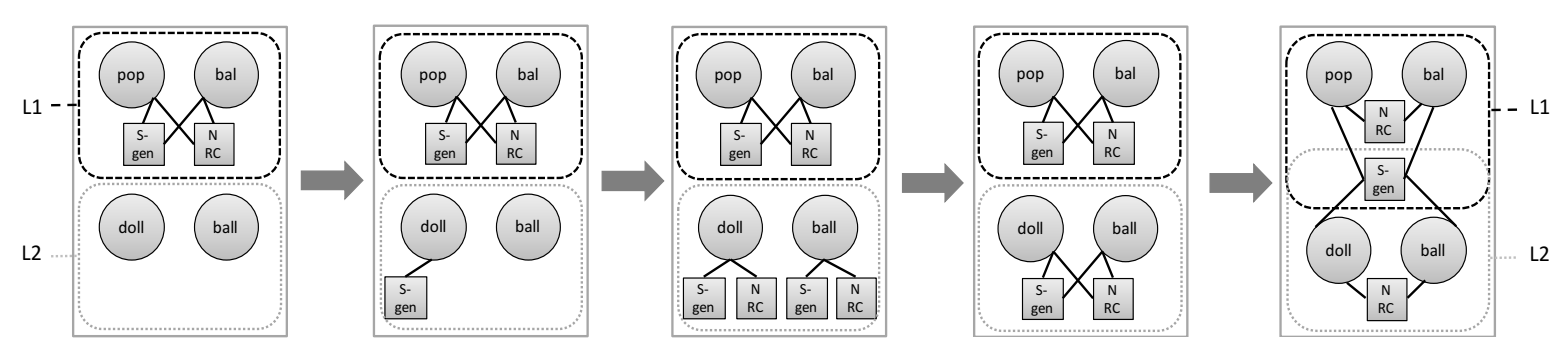

Figure 4. Developmental model. The L1 lexicon is depicted in the upper part of the model, the L2 lexicon in the lower part. To save space only lexical and combinatorial nodes are shown. pop and bal are nouns in L1 and doll and ball are verbs in L2; 'S-gen' and 'N RC' are combinatorial nodes. Consecutive model states during different points of the L2 syntactic acquisition trajectory are shown from left to right.

Panels 2, 3 and 4 show the development of syntactic structures in the L2. Panel two shows the stage that immediately follows after the initial contact with a new structure in the L2. We assume that, after a limited number of exposures, L2 learners start to form itemspecific representations of L2 syntactic structures. These representations can be structured, if they developed from lexical items (doll, ball) that got connected to a syntactic representation (s-genitive), but they can also represent formulaic expressions in the L2: Learners might, for example, know that "We did it!" indicates success, without knowing that the phrase contains two pronouns and a verb in the past tense. From the moment that a lexically specific syntactic representation is formed, learners will show lexically specific 
syntactic priming in L2 for this structure, even if prime and target structures don't immediately follow eachother, because the priming effects are now based on residual activation of lexically specific structural nodes or the strenghtening of syntactic procedures. The explicit memory of the prime structure can, however, still boost the production of the L2 structure, resulting in stronger priming in immediate conditions. As learners are no longer dependent on explicit memory for the production of the new L2 structure, they can produce it spontaneously, without being primed.

Panels 3 and 4 show how the process of abstraction takes place within the L2 lexicon. First, several lexically specific syntactic representations are added to the L2 lexicon, and then syntactic structures are formed that abstract across multiple words in the L2. Thus we assume that L2 syntactic representations develop separately from L1 syntactic representations in these stages, even though the representations in both lexicons might be quite similar. Based on only a few exposures to a structure with a particular lexical item the second language acquisition system cannot tell how item- specific this structure is in the L2: After encountering one English dative verb in a DO dative construction that is similar the Dutch DO dative construction, a Dutch learner of English cannot be sure whether this construction can be used for all other dative verbs as well. As the syntactic representations that are formed are still language specific, learners do not show between-language priming for these structures yet. When learners move from stage 3 to stage 4 , however, they will start to show lexically mediated and abstract syntactic priming in the $L 2$, as priming will now also result from residual activation of abstract structural representations. Since these representations are generalized across all lexical items they can be used with, learners will be able to use syntactic structures productively from now on, so also with lexical heads they have never perceived in combination with that specific syntactic structure. Note that this process of abstraction is driven by the type and token frequency of syntactic structures in the L2. Abstract syntactic representations will thus emerge earlier for more frequent structures than for structures that are less frequent. Consequently, it is possible that there is an intermediate state between the third and fourth state in our model, in which, for example, the s-genitive is already shared between 'doll' and 'ball', while there are still itemspecific representations for the post-modified noun phrase.

Finally, when they have reached a high level of proficiency in the L2, L2 learners get to the final stage of syntactic development, which is represented in panel 5 . The situation 
depicted here is a bit different from the situation depicted in the final panel of the model presented in Hartsuiker and Bernolet (2015). Here, we wanted to make clear that not all syntactic structures are shared eventually: L2 syntactic structures that have no corresponding syntactic structure in the L1 or that are not completely equivalent to their L1 counterpart may keep their language-specific representation in the $L 2$. We assume that that is the case for the representation of post modified nouns in English in the Dutch-English bilingual lexicon. In a study investigating within- and between-language syntactic priming for noun phrases (the red sheep vs the sheep that is red) in Dutch-English bilinguals, we found no between-language priming for these structures, while we did find within-language priming in Dutch and English. We attributed the absence of between-language priming to the word order difference in Dutch and English relative clauses (het schaap dat rood is - the sheep that is red): Because of this difference in word order, Dutch and English post modified nouns do not share a syntactic representation across languages. [The fact that we did obtain between-language priming between Dutch and German noun phrases, which use an identical word order for pre (het rode schaap - das rote Schaf) and post modified nouns (het schaap dat rood is - das Schaf das rot ist) lends support to this hypothesis.] Hence, in the last panel of the bilingual syntax model that is depicted here, only the s-genitive is represented in the part of the model that contains the shared syntax. This representation is connected to all nouns in the L1 (Dutch) and L2 (English) lexicon. For post modified noun phrases, the model contains a language-specific syntactic representation (N RC) in the $L 1$, which is connected to all nouns of the $L 1$, and a language-specific syntactic representation in the $L 2$, which is connected to all nouns of the $L 2$. Note, however, that we assume that structures are shared between languages as much as possible, so language-specific representations in the final stage of development should be the exception, rather than the rule. When structures are shared between languages, learners show equivalent syntactic priming within and between languages. Even in very proficient L2 users explicit memory mechanisms can co-determine syntactic choices. As we assume a larger role for explicit memory mechanisms when there is a large overlap between consecutive sentences, and because the overlap within languages is always larger than the overlap between languages, stronger within-language priming might be observed in conditions with related head nouns. 
With this model, we made a few specific claims about the development of $L 2$ syntactic structures in late second language learners. Firstly, we assume that, contrary to syntactic acquisition in L1, L2 acquisition in late learners begins with the learning of lexical representations without firm connections to abstract syntactic information. These itemspecific lexical representations become more abstract with increasing proficiency, with abstraction taking place across words within the $L 2$, and eventually also between languages. Our second claim is thus that, across the learning trajectory, L2 representations become more and more integrated with existing L1 representations. Consequently, syntactic processing and production in the L2 becomes more similar to L1 syntactic processing and production as learners become more proficient. Finally, we expect L1 influences on syntactic processing and production in the $\mathrm{L} 2$ to occur in two different phases in $\mathrm{L} 2$ syntactic development: In the earliest stages, when new syntactic structures of the L2 are first encountered, and in the final stage, when similar syntactic structures are fully shared between the L1 and the L2. In the following sections, we discuss evidence for these three claims.

\section{L2 syntactic representations in late learners move from being item-specific to being more} abstract

As we mentioned before, we assume that L2 learners start out with lexical representations of nouns and verbs, without firm connections to syntactic information. As no L2 syntactic information is available at this stage, learners have to rely on their knowledge of $L 2$ lexical items and L1 syntactic structures for the comprehension of novel sentences in the L2. For the production of a new syntactic structure L2 learners can use two different strategies: Transferring syntax from the L1 or imitating syntax from more competent speakers. The latter mechanism is based on the retrieval of sentences from explicit memory (see Bernolet, Collina \& Hartsuiker, 2016). Consequently, these L2 sentences, which are exact repetitions or minimally edited repetitions of earlier sentences, are only produced immediately after a more proficient speaker has used the 'example sentence'.

In the next stage of acquisition, item-specific representations of L2 structures start to emerge. As we will discuss below, several studies suggest that representations for new L2 syntactic structures can be formed after limited exposure to these structures (Kim \& 
McDonough, 2008; Nitchske, Kidd \& Serratrice, 2010). While the frequency of a specific verb/noun+structure combination determines how quickly an item-specific syntactic structure is represented in memory, the number of different verbs or nouns with which a specific structure is encountered determines how quickly an abstract representation of that structure is formed. Consequently, it is possible that specific verbs are initially only used with structure $X$, but not with structure $Y$ (second panel Figure 1), and that more frequent syntactic structures in the $L 2$ are abstracted across lexical items earlier than less frequently encountered structures. From the moment that a structure is generalized across lexical items, L2 learners can be primed within L2 to use the syntactic structure in conditions with and without lexical overlap. Eventually, they will be able to use the L2 syntactic structure productively, i.e. in unprimed conditions and with syntactic heads they have not necessarily encountered with this structure before.

Evidence for this abstraction process within the $L 2$ can be found in different studies investigating learning of English as a second language (ESL) by McDonough and colleagues (Kim \& McDonough, 2008; McDonough; 2006; McDonough \& Mackey, 2006). In 2006, McDonough and Mackey presented an intervention study that was carried out in a group of Thai university students studying English. The L2 learners were all selected to be at the same stage (stage 4) of Pienemann's developmental sequence for ESL question formation (Pienemann, Johnston \& Brindley, 1988, see McDonough \& Mackey for further information), so they had a comparable level of proficiency in the formulation of English questions. During the intervention, the L2 learners carried out a series of communicative tasks together with native speakers of English (three sessions in two weeks). These native speakers were either instructed to provide feedback on the learners' production in the form of recasts (responding with more targetlike forms whenever the learners produced nontargetlike question forms) or to abstain from giving feedback. Across 3 posttests (immediately after treatment and 3 and 6 weeks later), the results showed a significant effect of recasts on question development: While 23 out of 29 learners in the recast group had advanced to a higher stage in the developmental sequence for question formation, only 2 out of 19 learners in the no feedback group produced more advanced question forms.

Importantly, the investigators classified the learners' responses to the recasts in two different categories. Immediate and exact repetitions of the recast in the following turn (see example a, taken from McDonough \& Mackey, p. ) were discriminated from primed 
productions, in which the learner used the question form of the recast productively to form a new question within 6 turns of the recast (see example b). Further analyses showed that while primed production had a strong positive influence on the odds of development, the mere repetition of recasts in the next turn was not significantly correlated with ESL question development. We suggest that the repetitions could have been based on the retrieval of the recast from explicit memory: They were identical to the recast provided by the native speaker and immediately followed it. Consequently, the structure of these repeated questions did not necessarily have to be represented in memory. Primed productions of question forms, on the other hand, could only have been produced by learners who had already formed abstract representations of these question forms (because they were only called primed productions if they were new questions using the primed question structure), which is why only primed production during the intervention predicted the spontaneous production of advanced question forms at posttest. To summarize, this study shows that in the very early stages of acquisition learners can produce novel sentences in the $L 2$ even if they have no representation for the structure of these sentences by copying prime sentences that are explicitly recalled.

1. a) Repetition of a recast

[61] Learner: when it happen? Stage 3 question

[62] NS: when did it happen? Recast (stage 5)

[63] Learner: when did it happen? Repetition (stage 5)

b) Primed production (delayed)

[23] Learner: where where where you

Stage 3 question work this job?

[24] NS: Where did I work?

Recast (stage 5)

[25] Learner: yeah

[26] NS: I worked in America/it was my part time job during high school for three years

[27] Learner: why did you like it? Primed production (stage 5) 
In a different study, McDonough (2006) investigated the production of dative constructions in a within-language syntactic priming experiment with speakers of English as an L2. The participants in this study were advanced learners of English with a variety of different L1s (Chinese, Korean, Farsi, Portuguese, Serbian, Sinhala, Spanish, Thai and Turkish). The results of the first experiment in this study showed syntactic priming for the PO structure, but not for the DO structure. In fact, the DO structure was very infrequently used overall and when it was used, it was only in combination with a few verbs: The participants produced over half (63\%) of the double-object targets with only two verbs, ask and teach, and did not produce any double object targets with eight of the given verbs (bring, cook, cut, knit, make, pass, pour, and toss). For comparison: the same participants produced at least 8 PO datives with every single one of the 39 dative verbs in that experiment. According to McDonough, the DO dative is a developmentally more advanced form that is typically dispreferred by English L2 speakers. Consequently, it is possible that most of the $L 2$ learners in the study did not have an abstract L2 representation for the DO structure yet, leading them to only produce item-specific instances of this structure. In order to test whether increased exposure to DO datives would cause syntactic priming for these structures, a follow up experiment only used DO primes in the priming phase. The strong preference for the PO dative was not overturned, but the number of DO responses was significantly higher in the priming phase than in a pre-experimental baseline, suggesting that DO structures could be primed because abstract representations for this structure were formed. Thus this study is compatible with our claim that L2 learners start out with itemspecific syntactic representations in the $L 2$ and that the abstraction process from itemspecific to abstract representations in the $L 2$ is driven by the frequency of a specific structure and various verb/noun+structure combinations in the input, leading to earlier abstraction for more frequent structures.

Kim and McDonough (2008) provided additional evidence for the claim that in early stages of L2 syntactic acquisition syntactic processing relies on lexically specific representations rather than on abstract ones. In a study on transitive priming in English as a second language in a group of Korean-English L2 learners they showed evidence for structural priming as well as a lexical boost to priming. When they divided the participants in three groups of low, intermediate and high proficiency on the basis of a cloze test, they discovered that L2 proficiency was correlated with the strength of lexically based priming: 
All three groups showed stronger lexically based than abstract priming, but the difference between both conditions was largest in the low proficiency group. In fact, low proficiency learners hardly produced any passives when they had to use a different verb than the one used in the prime sentence, indicating that these participants still used lexically specific representations for this structure.

A final study that lends support to the claim that $L 2$ syntactic representations evolve from being lexically specific to more abstract was a study we did on syntactic priming of genitive structures (the wizard's guitar [s-genitive]/the guitar of the wizard [of-genitive]) in Dutch learners of English as an L2 (Bernolet, Hartsuiker \& Pickering, 2013). In one of both experiments in that study, we primed genitives within the $L 2$ of our participants. Before the experiment, participants rated their proficiency in English on a seven-point scale, so that we could investigate the effect of L2 proficiency on syntactic priming. Importantly, the experiment presented both types of genitive structures in related conditions, in which the head noun had to be repeated in the target structure (the boy's guitar/the guitar of the boy), and in unrelated conditions, in which the head nouns of prime and target constructions were unrelated (the boy's donkey/the donkey of the boy). This way, we could verify whether our participants showed lexically mediated as well as abstract priming for genitives in their $\mathrm{L} 2$. The less proficient bilinguals showed very strong lexically mediated, but hardly any abstract priming; more proficient bilinguals showed strong lexically mediated and abstract priming. Because the low proficient bilinguals mainly produced s-genitives in the repeated noun condition, when the prime construction could be almost completely copied to describe the target picture (as only the owner of the object differed), we concluded that these bilinguals might have several item-specific representations for this construction, instead of a representation that is generalized over different L2 nouns. Because the low proficient participants actually showed stronger priming than more proficient participants in the repeated noun conditions, we additionally assume that explicit memory processes might have played a bigger role in the lower proficiency participants' priming effects than in the priming effects observed for more proficient participants, who generated their responses from scratch.

2. Across the learning trajectory, $L 2$ representations become more and more integrated with existing $L 1$ representations 
As we mentioned, the final stage in our developmental model represents the situation that Hartsuiker et al. (2004) described in their model. In this final phase of development, abstract syntactic representations in the $L 2$ are merged with abstract syntactic representations in the $L 1$, if the representations are similar enough. From this moment on, the same syntactic representation or syntactic rule is activated and updated during processing in L1 and L2. Consequently, language-specific syntactic preferences may be merged into preferences that generalize across languages. As we will discuss in section 3, the activation of shared syntactic representations might also lead to transfer errors. In any case, complete syntactic sharing, which characterizes the final stage of our developmental model, leads to equivalent abstract syntactic priming within and between languages. Before syntactic structures are shared, however, between-language syntactic priming does not occur, leading to stronger priming within $\mathrm{L} 1$ and $\mathrm{L} 2$ than between these languages.

We are not going to discuss all the studies showing syntactic priming between languages in order to make a case for syntactic sharing in bilinguals, as this has been done in papers presenting earlier versions of our bilingual production model (Hartsuiker et al., 2004; Hartsuiker \& Pickering, 2008). Instead, it might be more interesting to focus on these studies which provide evidence for the claim that L2 learners use language specific strategies and representations for the comprehension and the production of L2 syntactic structures before they move on to the use of strategies and representations that are shared between their L1 and L2.

First of all, there are several neuroimaging and ERP studies which have shown increasing overlap between brain areas recruited for syntactic processing in L1 and L2 with increasing L2 proficiency (see Van Hell \& Tokowicz, 2010, for a review of ERP-studies and Van Heuven and Dijkstra (2010), for a review of neuroimaging data). The general pattern observed in most of these studies is that highly proficient bilinguals show native-like patterns for L2 processing, while low proficient bilinguals show very different patterns for both languages. In an fMRI study with late French-English bilinguals, for example, left prefrontal activation peaks - which the authors assumed to signal syntactic processing were found to be much closer during syntactic production L1 and L2 in more compared to less grammatically proficient bilinguals (Golestani, Alario, Meriau, Le Bihan, Dehaene \& Pallier, 2006). Thus this study suggests that $L 2$ learners only move to a stadium in which 
similar syntactic processing takes place in L1 and L2 after they have had sufficient exposure to the $L 2$ syntactic structures that are being learned. Before that moment, L2 specific representations guide comprehension and production in the $L 2$.

Evidence for the claim that L2 proficiency influences syntactic sharing was also found in a between-language syntactic priming experiment with Dutch and English genitive constructions (Bernolet et al., 2013). In this experiment, which was the between-language priming analog of the priming experiment discussed in the previous section, we used spoken Dutch genitive constructions to prime the use of English genitives (the boy's guitar [sgenitive]/the guitar of the boy [of-genitive]). Also in this experiment, both types of genitive structures were presented in conditions with related head nouns, in which the head noun of the target sentence was a translation equivalent of the head noun of the prime sentence (example $2 \mathrm{a} \& b)$, and in unrelated conditions, in which the head nouns of prime and target constructions were unrelated (example 2 c\&d). Overall, we obtained significant betweenlanguage priming for genitives and a translation equivalence boost to this effect: Stronger priming was observed in the related condition (23\% priming) than in the unrelated condition (6\% priming). More importantly, the priming effects in both conditions were modulated by participants' self-rated L2 proficiency: The more proficient the participants were, the stronger the priming effect. In fact, the participants with the lowest proficiency did not show any between-language priming at all, indicating that they had separate representations for English and Dutch s-genitives: Though the low proficiency participants sometimes used English s-genitives (mainly in the beginning of the experiment), the Dutch primes had virtually no effect on the response patterns.

2. TARGET: wizard holding a green guitar

a) De gitaar van de jongen is groen

of-genitive, related

[The guitar of the boy is green]

b) De jongen zijn gitaar is groen s-genitive, related

[lit. The boy his guitar is green (boy's guitar)]

c) De ezel van de jongen is groen of-genitive, unrelated [The donkey of the boy is green]

d) De jongen zijn ezel is groen s-genitive, unrelated

[The boy his donkey is green] 
Recently, we reanalyzed syntactic priming data of Schoonbaert et al. (2007) in order to verify whether the influence of $L 2$ proficiency on syntactic sharing could be generalized to other structures (in this case L2 datives) as well (Hartsuiker \& Bernolet, 2016). English genitives are quite hard to learn for Dutch-English bilinguals like to ones who participated in our 2013 study (Bernolet et al., 2013), as the spoken form of the Dutch s-genitive differs from its English counterpart in terms of morphological realization: Whereas the Dutch genitive uses a possessive pronoun that takes the gender and the number of the posessor it refers to (zijn [his]/haar [her]/hun [their]), the English variant uses the same bound morpheme ('s) for all nouns and possessors it combines with (see examples 2 b \& $d$ above). This difference in the realization of the possessive element increases the risk of making transfer errors (e.g. the nurse her shoe is green was one of the 42 transfer errors observed in our between-language priming experiment), which might lead to a longer learning trajectory for English genitives than for structures like datives, for example, where the Dutch and English variants are literal translations. As Dutch-English bilinguals might need less exposure to English dative structures to go through all of the developmental stages in our model, we hypothesized that effects of $L 2$ proficiency on between-language priming would be smaller or even non observable in Schoonbaert et al.'s (2007) data on betweenlanguage priming for datives. Nevertheless we found clear effects of L2 proficiency: In the related condition, in which the head verbs of prime and target constructions were translation equivalents (geven-give), as well as in the unrelated condition, the betweenlanguage priming effects increased together with the participants' self-rated proficiency in their L2. Even though the DO and the PO dative are much more similar between Dutch and English than of- and s-genitives, lower proficiency L2 speakers do not seem to have abstract, language-independent representations yet for this structure. Thus, the results of our reanalysis of the Schoonbaert et al. (2007) data suggest that the proficiency effects in the production of English genitives are not structure-specific, but rather reflect a strategy by which new syntactic representations in L2 are are kept separated from their L1 counterparts in the early stages of $L 2$ syntactic acquisition.

Finally, a study by Runnqvist, Gollan, Costa, and Ferreira (2013) provided evidence for the claim that the evolution from language-specific to shared syntactic representations entails that language-specific syntactic preferences are merged into preferences that 
generalize across languages. In this study, response times for the initiation of English transitives and genitive constructions were measured for three different groups of participants: English monolinguals, Mandarin-English bilinguals, and Spanish-English bilinguals. Crucially, the genitive constructions that were used in their study (the woman's stroller is pink/the stroller of the woman is pink) have different relative frequencies in Spanish, Mandarin, and English. In English, both alternatives are used for the possessive, but pre-modified possessive NPs (s-genitives) are more frequent than post-modified possessive NPs (of-genitives). In Mandarin, however, possessive NPs are always pre-modified, while in Spanish all non-pronominal possessive NPs are post-modified. The English monolinguals in this study were faster to initiate pre-modified NPs than post-modified NPs. This frequency effect was significantly larger in English for Mandarin-English bilinguals, but not for SpanishEnglish bilinguals, a pattern that can only be explained by assuming that the frequencies of syntactic structures accumulate across languages: The Mandarin pre-modified NP boosts the frequency of the structure that is already more frequent in English, but not that of the competing post-modified NP, leading to a larger frequency effect. In Spanish, the postmodified NP adds to the frequency of the structure that is least frequent in English, bringing the frequencies of both constructions closer together and minimizing the frequency effect.

To summarize, several studies have shown that bilinguals evolve from using specialized memory representations and procedures to comprehend and produce L2 syntax to using the same representations and procedures for syntactic processing in L1 and L2. Because in this final stage the same memory representations are activated during processing in $\mathrm{L} 1$ and $\mathrm{L} 2$, sentence comprehension and production shows generalized priming (equally strong priming within and between languages) and frequency effects.

\section{L1 influences on syntactic processing and production in the L2 occur in the earliest phase} as well as in the final phase of L2 syntactic development

As we already mentioned, we assume that learners rely on their combined knowledge of L2 lexical items and L1 syntactic structures for the comprehension of novel sentences in the L2, because in the initial stage of L2 syntactic acquisition, only lexical representations of L2 nouns and verbs are avaliable. In this initial stage, it is not unlikely that L1 syntactic representations are used for the comprehension and the production of $L 2$ sentences. When 
the L1 and the L2 are closely related (like, for example, Dutch and English) syntactic transfer from the L1 will often lead to correct interpretations and productions. When the L1 and L2 structures are not completely identical, however, this kind of transfer leads to a syntactic error. We assume that these kinds of errors might trigger the separation between L1 and L2 syntactic representations in the following stages of acquisition. After a learning phase in which transfer errors don't occur, because separate memory representations are used for L1 and L2 syntactic processing, transfer errors might pop up again as a consequence of syntactic sharing. In this case, it is not the general syntactic structure that is transferred, however, because this is assumed to be shared between languages. Rather, the error arises because language-specific features of a shared syntactic structure are transferred between languages. In any case, we assume that L2 syntactic development is characterized by early and late transfer errors, with an errorfree phase in between.

Evidence for early transfer of L1 syntax is provided in a study by Nitschke, Kidd \& Serratrice (2010), which investigated L1 transfer and syntactic priming through comprehension in L1 and L2 speakers. The critical structures used in this study were German and Italian RC structures that are ambiguous between subject and object interpretations (German: Hier ist die Frau, die das Mädchen küsst; Italian: Ecco la donna che bacia la ragazza), but which are structurally identical to unambiguous RC structures in English (object RC: Here is the woman that the girl kisses; subject RC: Here is the woman that kisses the girl). These structures allowed the researchers to investigate whether there is an influence of L1 syntactic preferences in the comprehension of L2 German and Italian: The preferred reading of both the German and the Italian sentence is one in which the woman is assigned the subject role. Because an SOV reading of the German sentence is structurally not possible in English, however, the researchers expected a higher preference for the object reading (the girl kisses the woman) in English-German bilinguals than for native Germans. For the Italian sentences they predicted low proportions of object readings for native Italians and English-Italian bilinguals, as an SVO reading is preferred both in English and in Italian. The results of a picture selection task indicated that the English-German participants had a higher preference for the object reading than all other participant groups, indicating that L1 English syntactic preferences influenced the comprehension of L2 German sentences. A priming phase in which participants were only primed with sentences that allowed an object reading resulted in increased proportions of object readings for German 
L1s, English-German bilinguals and English-Italian bilinguals in the priming phase and in a posttest. This indicates that, while the L1 syntactic preferences initially guided comprehension, these preferences were overridden by increased exposure to L2 syntactic structures.

As mentioned above, we found many L1 transfer errors in our study on within- and between-language priming of English genitives (Bernolet et al. 2013). Errors like the nurse her shoe is green, in which a possessive pronoun is used in an English s-genitive, occurred more frequently in our between-language priming experiment ( $4 \%$ of all responses) than in our within-language priming experiment (2\% of all responses). Because - especially in our between-language priming experiment- the majority of s-genitives was produced by more proficient participants, most of the transfer errors were also produced by more proficient participants. Transfer errors were more frequent in same meaning conditions than in conditions with unrelated heads and they often occurred in contexts where, in Dutch, adding an s-morpheme would have to be avoided (the nurse's shoe contains three sibilants in a row). This led us to conclude that these errors might be a side effect of having a shared syntactic representation for Dutch and English s-genitives: If the same syntactic node is activated during the processing and the production of Dutch and English s-genitives, there is a risk that the morpho-syntactic rules for the formation of the Dutch s-genitive 'intrude' into the s-genitive that is used to describe the target picture.

Further evidence for late transfer errors or co-activation errors is provided in two ERP-studies by Thierry and Sanoudaki (2013) and Vaughan-Evans, Kuipers, Thierry and Jones (2014). Thierry and Sanoudaki (2013) compared the comprehension of adjective-noun and noun-adjective pairs in English between English monolinguals and native Welsh-English bilinguals. The results of their experiment indicated that the bilinguals expected an adjective following a noun in an English sentence, even though this word order is illegal in English. In Welsh, however, this word order can be used, alongside the adjective-noun order which is used in English. Thierry and Sanoudaki (2013) concluded that Welsh-English bilinguals might have shared representations for the modification of nouns in Welsh and English, which led them to expect an erroneous word order in English, in contrast to the monolingual participants, who only expected adjective-noun combinations. Vaughan-Evans, Kuipers, Thierry and Jones (2014) showed intrusions of Welsh syntax in the comprehension of English syntax by presenting Welsh-English bilinguals with English sentences that ended in 
nonwords. The nonwords were English words (e.g. patients) of which the intial consonant was either aberrant (e.g. datients) or followed a Welsh mutation rule (e.g. batients). The results of the experiment indicated that English words that were mutated according to Welsh mutation rules were more easily integrated into the sentence context (they showed a smaller phonological mismatch negativity) when they were presented in a context that warranted the rule in Welsh than in a nonmutation context.

The two studies mentioned above both studied native or very early Welsh-English bilinguals. In these bilinguals, the syntactic representations developed simultaneously in the languages they know. Hence, our developmental model might not capture the syntactic development these Welsh-English bilinguals went through. What we can say, is that, even as native bilinguals, they ended up with syntactic representations that are fully shared between languages. Hence, these studies show that, even for bilinguals with a very high proficiency in both of their languages, syntactic sharing can lead to the production of erroneous syntax, or non-native-like processing strategies.

\section{General discussion}

In the previous sections, we sketched a possible account of $L 2$ syntactic acquisition in late learners (Hartsuiker \& Bernolet, 2016) and discussed the studies on which the core assumptions of this account are based. On the basis of the studies that were discussed, it seems quite plausible to assume that the L2 syntactic representations evolve in the way that is put forward in the model: There is evidence for an abstraction process within the L2, for more syntactic sharing with increasing proficiency, and for early and late co-activation of L1 syntactic rules during L2 syntactic processing. The problem is, however, that none of the studies discussed above tested participants through the complete learning trajectory of a specific syntactic structure in the L2. Additionally, it is difficult to compare the L2 proficiency of the L2 learners across studies. Consequently, we cannot be sure that L2 learners go through all of the 5 stages proposed in the model. Studies with longitudinal designs that follow participants from their first encounter with a syntactic structure in the $L 2$ until the moment that the syntactic structure is completely mastered could shed light on this.

Another shortcoming of Hartsuiker and Bernolet's (2016) model, is that the learning mechanisms are underspecified. As we mentioned, the process of abstraction within the L2 and, eventually, across the L1 and the L2 could be driven by a form of Hebbian learning - if 
two nodes are always active together they might become functionally equivalent to a single node. The general principles of specificity and economy we assume are, however, also compatible with distributed theories (e.g. Chang et al., 2006) which assume error-based learning of syntactic rules. Both learning principles make different predictions concerning the strenth of priming: Whereas Hebbian learning causes stronger priming for structures that are more frequent, error-based learning predicts the opposite pattern. A comparison of within- and between-language priming caused by L1 and L2 syntactic primes might help to clear out this issue.

To conclude, we have proposed and discussed a possible account of L2 syntactic acquisition in late learners. More research is needed to refine our account of what happens during the late learning of L2 syntax, and what learning mechanisms are involved. 
List of references

Bernolet, S., Collina, S., \& Hartsuiker, R.J. (2016). The persistence of syntactic priming revisited. Journal of Memory and Language, 91, 99-116.

Bernolet, S., Hartsuiker, R. J., \& Pickering, M. J. (2007). Shared syntactic representations in bilinguals: Evidence for the role of word-order repetition. Journal of Experimental Psychology: Learning, Memory, and Cognition, 33, 931-949.

Bernolet, S., Hartsuiker, R.J., \& Pickering, M.J. (2011). Effects of phonological feedback on the selection of syntax: Evidence from between-language syntactic priming. Bilingualism: Language and Cognition, 15, 503-516.

Bernolet, S., Hartsuiker, R. J., \& Pickering, M. J. (2013). From language-specific to shared syntactic representations: The influence of second language proficiency on syntactic sharing in bilinguals. Cognition, 127, 287-306.

Cai, Z. G., Pickering, M. J., Yan, H., \& Branigan, H. P. (2011). Lexical and syntactic representations between closely related languages: Evidence from Cantonese-Mandarin bilinguals. Journal of Memory and Language, 65, 431-445.

Chang, F., Dell, G. S., \& Bock, K. (2006). Becoming Syntactic. Psychological Review, 113, $234-272$.

Cleland, A. A., \& Pickering, M. J. (2003). The use of lexical and syntactic information in language production: Evidence from the priming of noun-phrase structure. Journal of Memory and Language, 49, 214-230.

Golestani, N., Alario, F. X., Meriaux, S., Le Bihan, D., Dehaene, S., \& Pallier, C. (2006). Syntax production in bilinguals. Neuropsychologia, 44, 1029-1040.

Hartsuiker, R., \& Bernolet, S. (2016). The development of shared syntax in second language learning. Bilingualism: Language and Cognition. doi:10.1017/S1366728915000164 
Hartsuiker, R. J., Pickering, M. J., \& Veltkamp, E. (2004). Is Syntax Separate or Shared Between Languages? Cross-Linguistic Syntactic Priming in Spanish-English Bilinguals. Psychological Science, 15, 409-414.

Hartsuiker, R.J., \& Pickering, M.J. (2008). Language integration in bilingual sentence production. Acta Psychologica, 128, 479-489.

Kantola, L., \& van Gompel, R. P. G. (2011). Between- and within- language priming is the same: Evidence for shared bilingual syntactic representations. Memory \& Cognition, 39, 276-290.

Kim, Y., \& McDonough, K. (2008). Learners' production of passives during syntactic priming activities. Applied Linguistics, 29, 149-154.

McDonough, K. (2006). Interaction and syntactic priming: English L2 speakers' production of dative constructions. Studies in Second Language Acquisition, 28, 179-207.

McDonough, K., \& Mackey, A. (2006). Responses to recasts: Repetitions, primed production, and linguistic development. Language Learning, 56, 693-720.

Nitschke, S., Kidd, E., \& Serratrice, L. (2010). First language transfer and long-term structural priming in comprehension. Language and Cognitive Processes, 25, 94 - 114.

Pickering, M. J., \& Branigan, H. P. (1998). The representation of verbs: Evidence from syntactic priming in language production. Journal of Memory and Language, 39, 633651.

Pienemann, M., Johnston, M., \& Brindley, G. (1988). Constructing an acquisition-based procedure for second language assessment. Studies in Second Language Acquisition, $10,217-243$.

Runnqvist, E., Gollan, T. H., Costa, A., \& Ferreira, V. S. (2013). A disadvantage in bilingual sentence production modulated by syntactic frequency and similarity across languages. Cognition, 129, 256-263. 
Salamoura, A., \& Williams, J. N. (2006). Lexical activation of cross- language syntactic priming. Bilingualism: Language and Cognition, 9, 299-307.

Salamoura, A., \& Williams, J. N. (2007). Processing verb argument structure across languages: Evidence for shared representations in the bilingual lexicon. Applied Psycholinguistics, 28, 627-660.

Schoonbaert, S., Hartsuiker, R. J., \& Pickering, M. J. (2007). The representation of lexical and syntactic information in bilinguals: Evidence from syntactic priming. Journal of Memory and Language, 56, 153-171.

Shin, J. A., \& Christianson, K. (2009). Syntactic processing in Korean-English bilingual production: Evidence from cross-linguistic structural priming. Cognition, 112, 175180.

Shin, J. A., \& Christianson, K. (2012). Syntactic processing in Korean-English. Cognition, $112,175-180$.

Thierry, G., \& Sanoudaki, E. (2012). Activation syntaxique non-sélective à la langue chez le bilingue précoce. Revue Française de Linguistique Appliquée, XVII-2, 33-48.

Van Hell, J. G., \& Tokowicz, N. (2010). Event-related brain potentials and second language learning: Syntactic processing in late L2 learners at different L2 proficiency levels. Second Language Research, 26, 43-74.

Van Heuven, W. J. B., \& Dijkstra, T. (2010). Language comprehension in the bilingual brain: fMRI and ERP support for psycholinguistic models. Brain Research Reviews, 64, 104 122.

Vaughan-Evans, A., Kuipers, J.R., Thierry, G., \& Jones, M.W. (2014). Anomalous Transfer of Syntax between Languages. The Journal of Neuroscience, 34, 8333-8335. 
